\title{
Context and Strategy in Acquiring Temporal Connectives ${ }^{1}$
}

\author{
Deborah Keller-Cohen ${ }^{2}$
}

Accepted August 8, 1986

There has been considerable debate over the role of comprehension strategies in the acquisition of temporal connectives. This study examined the role of clause logic and interpretational strategies in the acquisition of temporal words; age-related changes were also considered. Thirty-two children between 3 and 5 years of age served as subjects. Sentences with a variety of temporal words were used to tap children's comprehension of "before," "after," "when," "while," "just before that," and "and after that." Clause logic was found to significantly improve the understanding of these sentences. However, the order of mention and main clause first strategies were used infrequently. Apparently, when children respond to sentences that describe information in a way that is consistent with what they normally hear, these strategies are seldom applied.

There has been considerable interest in recent years in how children learn the meanings of sentences with temporal connectives, mainly before and after (Clark, 1971; Johnson, 1975; Trosborg, 1982). A recurrent theme in this research is the way children approach interpreting these sentences when they have not yet fully mastered their meaning. One difficulty in evaluating this has been that past approaches have not always looked at the relationship between age and the use of strategies for interpreting sentences with temporal connectives. Thus, it has not been clear whether the frequency and type of strategy change with age.

\footnotetext{
${ }^{1}$ I wish to express my thanks to Janice Bogen, who assisted in data collection, and to Erika Hoff-Ginsberg, who assisted in some of the data analysis.

${ }^{2}$ Address all correspondence to Deborah Keller-Cohen, English Composition Board, The University of Michigan, Ann Arbor, Michigan 48109.
} 
Furthermore, it has not always been possible to pull apart the effects of temporal connective and strategy-that is, whether the use of a particular comprehension strategy is triggered by a particular connective. The present study assesses how the use of comprehension strategies is affected by age, the semantics of a wide range of temporal connectives, and the meaning of the clauses joined by the connectives. In this respect, the present investigation extends research on the role of contextual cues (clause logic) in learning the meaning of words (French \& Brown, 1977) and the contribution of age-related strategies to interpreting temporal terms (Kavanaugh, 1979).

\section{Clause Logic}

The use of clause logic to interpret temporal connectives can be evaluated in several ways. One tack has been to compare contexts whose logic supports the meaning of such terms, as in 1, with those that do not, as in 2.

1. The boy opened the can before he poured the soup. (LOGICAL RELATIONSHIP)

2. The girl ate the cake after she opened the door. (ARBITRARY RELATIONSHIP)

This is the method utilized in previous studies of some temporal terms (Kavanaugh, 1979; Trosborg, 1982). A second approach would be to look more closely at the effect of clause logic on the acquisition of particular connectives to discover whether some terms are aided by clause logic more than others. Here we would ask whether connectives whose meaning may be reinforced by clause content, such as before in number 1 above, are learned earlier than those whose meaning is not (e.g., while in He opened the can while he drank the pop). The effect of clause logic also can be explored with multiple-sense connectives, such as when, whose interpretation may depend upon the logical relationship between the events described in the clauses as in 1 (logical) or 2 (arbitrary) above. These three approaches are considered in greater detail below.

There has been a good deal of evidence for the view that children first learn the meaning of some temporal terms in sentences where the clause provides support for interpreting these terms. For example, French and Brown (1977) examined children's interpretations of sentences where the events in the clauses formed a logical series, Raggedy Ann fills the bottle before she feeds the baby, and others where the events were arbitrarily related, Raggedy Ann goes to bed after the dog runs away. 
They found that performance on logical sequences was superior to that on arbitrary sequences. (see also Trosborg, 1982, for similar results.) This suggests that the children may have been using the relationship between the clauses to interpret such sentences. In this view, a child might have partial knowledge of the temporal terms, and the presence of clauses describing logically ordered events might be used to provide decisive information about the meaning of the connectives. However, the possibility exists that a child may have been relying on his world knowledge about the relation between the clause events to understand the sentences, not processing the connectives at all. Prior work does not provide a means of deciding between these alternatives.

One way to show that a child is relying on his extralinguistic knowledge alone to process a sentence with logically related sequences is to observe his responses to sentences with events in reverse of their logical order, as in 3 and 4.

3. After the boy poured the soup, he opened the can.

4. The boy ate the hot dog and after that he poured the ketchup.

If a child is relying on the logical order of events to form his interpretation of these sentences rather than on knowledge of the connectives or on a combination of both, he should dramatize these sentences incorrectly, selecting as the first event the one that occurs first in a real-world series (e.g., The boy opened the can).

Now let us consider the reverse logical sequence in relation to the arbitrary and logical sequences used in previous work (French \& Brown 1977; Kavanaugh, 1979). If a child does not know the meaning of any temporal connectives and relies solely on his knowledge of event relationships in the world to interpret these sentences, he should interpret correctly all logical sequences and interpret incorrectly both reverse logical and arbitrary sequences. As he begins to learn the meaning of some connectives, presumably in logical sequences, he may still rely primarily on the clause relationship to assign a meaning to such sentences; however, where this is not possible (arbitrary sequences), he should begin to draw on his knowledge of the connectives. This would result in his responding correctly to logical sequences, failing many reverse logical sequences and giving some correct responses to arbitrary sentences. Finally, a child would take both clause content and the temporal connective into account. This set of predictions is similar to that of French and Brown (1977) but extends their model by including responses to reverse logical sequences in order to clarify when the clause 
logic is being relied on to interpret these sentences to the exclusion of the temporal connective in the sentence.

An additional role of clause logic in the acquisition of temporal connectives can be seen by comparing terms whose meaning can be learned by utilizing such clausal information (such as before, after, when) with a term that tends not to be (while). In particular, we would expect children to learn the meaning of contextually supported words (before, after) prior to that of while where the clause context provides little assistance. (See Ferreiro, 1971, for related work.)

As the child learns more about the meaning of temporal connectives and about the role clause logic plays in the interpretation of sentences, we should begin to see him utilize this knowledge in new ways. The acquisition of a multiple-sense term such as when affords us a unique opportunity to observe children's use of clause logic to decide between alternate interpretations of a single word. For example, when may mean "after" as in 5 and 6, or either "after" or "while" as in 7.

5. When the boy opened the can, he poured the soup.

6. The boy drank the juice when he opened the bottle.

7. The boy ate the pancakes when he opened the soup.

If a child uses the clause logic to interpret when, he ought to respond differently to sentences where the clauses are logically related (5 or 6) than to sentences where they are not (7). In this view, a child knows the senses of a term and recognizes that the logic of clauses should be used to select one reading over another.

A second issue in the literature is the role of particular strategies in children's interpretations of sentences with temporal connectives. The use of clause logic to the exclusion of the temporal connectives in the sentences is one strategy that has been suggested above. Two other heuristics continue to be reported: an order of mention strategy and the main clause first strategy. These are described below.

\section{ORDER OF MENTION}

Most studies of temporal reference report that some children use an order of mention strategy to interpret sentences with temporal connectives (Bever, 1970; Clark, 1971; Johnson, 1975). In using the order of mention strategy, some children interpret sentences with temporal connectives by treating the order in which the events are mentioned as the order in which the events occurred. For example, in a comprehension task Clark (1971) 
found that 3 -year-olds treated sentences 8 and 9 identically, dramatizing the boy kicked the rock first. She also observed that this strategy decreased with age.

8. The boy kicked the rock before he patted the dog.

9. Before the boy kicked the rock, he patted the dog.

\section{MAIN CLAUSE FIRST}

Still other work reports that children use a main clause first strategy to process sentences with a connective. The main clause first strategy is just that - the child processes the main clause first. Three response patterns have been taken as evidence of this strategy: (a) Children ignore the event in the subordinate clause and do not dramatize it on a comprehension task (Amidon \& Carey, 1972; French \& Brown, 1977); only the main clause event is acted out; (b) children dramatize the main clause first and the subordinate clause second (Coker, 1978); (c) children correctly interpret sentences with the main clause first more often than those with a subordinate clause first (Keller-Cohen, 1974).

\section{INTERACTIONS BETWEEN STRATEGIES AND OTHER VARIABLES}

Prior work has made significant strides in relating many of the interpretational strategies described above and task variables. However, important questions remain regarding developmental changes during the preschool years in the use of these strategies and in their interaction with the logic between events described in the clauses. It has been difficult to assess the developmental changes in the role of these strategies in part because of a potential confounding of the particular temporal connectives studied with interpretational strategies. For example, consider the following sentence frames: (a) event 1 before event 2; (b) before event 2, event 1 ; (c) after event 1 , event 2; (d) event 2 after event 1.

A child who understands the meaning of before but not after would correctly interpret sentence frames (a) and (b) but not (c) and (d). However, this same response pattern would be found for children who process and dramatize the main clause before the subordinate clause (Coker, 1978). The present investigation addresses this problem by examining a wider range of temporal constructions, specifically before, after, but before that, and after that, when, and while. Questions also 
remain about the changes with age in the role of both the order of mention and main clause interpretational strategies (Kavanaugh, 1979). This issue is examined here in four groups of preschool children from 3 to 5 years of age.

Finally, the present study investigates the relationship between clause logic and interpretation strategy. This is accomplished by examining the use of the strategies cited above in logical and in arbitrary sequences.

\section{METHOD}

\section{Subjects}

Thirty-two children from the Ann Arbor and Buffalo areas served as subjects: 19 females and 13 males. The subjects were partitioned into four groups of eight children each: Group I (3 years 1 month-3 years 5 months), Group II ( 3 years 6 months - 3 years 11 months), Group III (4 years -4 years 5 months), and Group IV (4 years 6 months -4 years 11 months).

\section{Materials}

Children's interpretation of sentences with temporal connectives was measured by asking them to use toys to dramatize sentences read aloud. The toys were a boy and a girl doll and doll-sized objects such as tiny cans of soup, cake, keys, and bottles. In addition, a doll-sized door was constructed and attached to a frame so that a doll could be made to open a door.

\section{Instrument}

Each test consisted of 52 sentences. Each sentence consisted of a two-event sequence and there were three types of sequences: logical, reverse logical, and arbitrary. The logical sequences were those in which the events occurred in a predictable but noncausal order. For example, in The girl poured the syrup before she ate the pancakes the sequence is one we often witness but the eating of pancakes is not the result of pouring the syrup. Reverse logical sequences were those in which the event order was counter to what one would expect. For example, we normally blow out birthday candles before we cut the cake. Thus, After the girl cut the cake, 
she blew out the candles reverses this order. In arbitrary sequences, two events that have no predictable temporal relationship are described, as in The boy opened the door after he ate the pancakes.

Eight constructions (types 10-17 below) used twice formed the 16 sentences for the logical and the 16 sentences for the reverse logical order conditions; 10 constructions (10-19 below) used twice formed the sentences for the arbitrary order condition. Due to a clerical error, one occurrence of but before that was omitted from the logical order condition, leaving 15 rather than 16 sentences in that condition only. Ten constructions were used:

10. $X$ before $Y$

11. After X, Y

12. $X$ and after that $Y$

13. When $X, Y$

14. Before $X, Y$

15. $\mathrm{Y}$ after $\mathrm{X}$

16. $\mathrm{Y}$ but before that $\mathrm{X}$

17. $Y$ when $X$

18. $X$ while $Y$

19. While $X, Y$

For purposes of analysis, these 10 constructions were collapsed into three categories that described the semantic relationships expressed by the following constructions: Simple Sequence $(10,11,12)$, where events are described in their order of occurrence; Reverse Sequence $(14,15,16)$, where events are described in reverse of their order of occurrence; and Simultaneity $(18,19)$, where events are described that cooccur. Sentences with when (13 and 17) can be used to describe either sequential or simultaneous events. In the sequential reading, 13 describes simple sequence and 17 reverse sequence. In their simultaneity reading, 13 and 17 describe cooccurring events.

The first eight constructions appeared in all three logic conditions. Sentences with while appeared in the arbitrary order condition only since while does not sequentially order events.

\section{Design}

Four randomizations (versions) of the 52 test sentences were prepared. Each version was divided into two parts, A and B. This resulted in eight versions: version $1 \mathrm{AB}$, version $1 \mathrm{BA} \ldots$ version $4 \mathrm{BA}$. An equal number of subjects $(n=4)$ received each of the versions. 
The overall design initially included six independent variables: age, version, and order in which the version half was presented were between-subject variables; clause logic (logical, reverse logical, arbitrary), temporal relationship (simple sequence, reverse sequence, and simultaneity), and subordinate clause position were within-subject variables.

\section{Procedure}

A female experimenter tested each child separately. After several minutes of casual conversation, the experimenter demonstrated each of the events a child would hear in the stimulus sentences. Then the child was asked to dramatize each of these events; no child had any difficulty learning these dramatizations. Indeed, the children were uniformly delighted with the minature objects and eager to have the opportunity to use them. After this preliminary period the experimenter began the test, reading each sentence only twice. In the event that a child became distracted, the experimenter returned to the sentence at the end of the test.

\section{RESULTS}

Preliminary analyses were aimed at determining the effect of the test version and the effect of the order in which the version half was presented. A repeated-measures ANOVA revealed no main effect for version, $F(3,24)=1.32$, or half order, $F(4,24)=3.62$. Thus, these two variables were excluded from subsequent analyses, leaving four variables remaining, (age, clause logic, temporal relationship, and subordinate clause position).

The first stage of the analysis was aimed at examining children's use of clause logic to understand sentences with temporal connectives. The investigation consisted of three steps. First, we explored whether the logical relationship between events in two clauses influenced children's interpretation of temporal connectives. If this was so, sentences with clauses describing events in a predictable sequence (The girl opened the can; She poured the soup) should be easier to understand than those where the events do not occur in a predictable order (The boy picked up the key; He ate the pancakes). All temporal connectives except when were included in this analysis. Responses to when were analyzed separately because the opportunity for a correct response to sentences with when was greater since either an "after" or a "while" interpretation was considered acceptable. The second step in examining the effect of clause logic was to compare performance on sentences with connectives 
Table I. Proportion of Correct Responses by Age ${ }^{a}$

\begin{tabular}{cccc}
\hline & \multicolumn{3}{c}{ Clause logic } \\
\cline { 2 - 4 } Age group & Logical & Reverse logical & Arbitrary \\
\hline I & .88 & .34 & $.48^{b}$ \\
II & .64 & .49 & $.61^{c}$ \\
III & .86 & .60 & .57 \\
IV & .93 & .72 & $.80^{d}$ \\
$\bar{X}$ & .83 & .54 & .62 \\
\hline
\end{tabular}

${ }^{a}$ Excluding sentences with when.

Comparisons between Reverse Logical and Arbitrary orders:

${ }^{b} p<.005$.

$c_{p}<.025$.

${ }_{p}<.050$.

whose meaning is reinforced by clause logic (e.g., before in The girl opened the can before she drank the juice) with those where the meaning of the connective is not (while). The third part of this analysis focused on the role clause logic plays in the interpretation of a connective with multiple meanings. In particular, we wondered whether children's interpretation of when depended on the logic of the clauses with which it occurred. For example, we explored whether they were more likely to interpret when as after rather than as while in the following sentences: The girl ate the hot dog when she poured the ketchup; When the boy opened the box, he poured the cereal.

As predicted, there was a main effect for clause logic, $F(2,56)=$ $41.55, p<.001$. The proportion correct was greatest on logical order sentences $(83 \%)$ and considerably worse on arbitrary order $(62 \%)$ and reverse logical order sentences (54\%). In Groups I, II, and IV performance on arbitrarily related events was significantly better than on reverse logical events, although the magnitude of difference decreased with age. The number of correct responses on all three categories increased with age, $F(3,28)=4.02, p<.05$. This is summarized in Table I.

The influence of clause logic can also be seen in the children's performance on contextually unsupported while as compared to the connectives (before, but before that, after, and after that) that may receive contextual support. We compared the frequency of correct responses to each of these constructions in the arbitrary clause relation condition. Sentences in this condition only were used since while did not 
Table II. Proportion of "After" and "While" Interpretations of When ${ }^{a}$

\begin{tabular}{crcc}
\hline \multirow{2}{*}{ Age group } & & \multicolumn{3}{c}{ Clause logic } \\
\cline { 3 - 4 } & & Logical & Arbitrary \\
\hline \multirow{2}{*}{ I } & "After" & .72 & .53 \\
& "While" & .09 & .03 \\
II $\quad$ "After" & .78 & .59 \\
& "While" & .06 & .09 \\
III & "After" & .78 & .59 \\
& "While" & .06 & .13 \\
IV & "After" & .59 & .53 \\
& "While", & .22 & .38 \\
\hline
\end{tabular}

"The "after" and "while" responses do not sum to 1.00 because children produced other responses to when sentences. These include omissions of one of the clauses and "before" or reversal responses.

appear in the natural or reverse natural order conditions. If clause logic helps children learn the meaning of temporal connectives, we would expect children to learn first terms whose meaning is reinforced by clause logic (before, after) and later master terms that are not (while). A repeated-measures ANOVA revealed a main effect for temporal construction $F(2,56)=34.62, p<.001$. Children produced a significantly greater number of correct responses on all constructions with before and after than on sentences with while, $T(31)=7.06, p<.001$.

Responses to when provided additional information about the effect of clause relationship. An analysis of variance revealed that clause logic significantly affected interpretations of when, $F(2,56)=13.13, p<$ .001. A closer look at this appears in Table II.

"After" interpretations of when were more frequent in the logical than in the arbitrary condition; this pattern is stable until 5 years of age, where the difference nearly disappears. This decrease may be due in part to the acquisition of the "while" sense of when, which begins to be more frequent at age 5, undoubtedly replacing some "after" interpretations. A "while" reading of when accounted for few responses until 5 years of age, when it became more frequent in the arbitrary than in the logical condition.

\section{Interpretational Strategies}

One of the central questions in this study was how children modify their strategies for interpreting temporal connectives as they grow older. 
The next set of analyses examined changes in three interpretational strategies: Logical Order, Order of Mention, and Main Clause First.

Each child's responses were scored for evidence of each of these strategies. As in French \& Brown (1977), a child was considered to use a strategy if the majority ( $75 \%$ or more) of his responses corresponded to the criteria described under a particular strategy outlined below. The scoring procedures were as follows:

\section{Logical Order Strategy}

The data for this analysis were responses to all sentences where the event pairs could be thought of as having a logical order whether or not they were described in that order. Thus, reverse logical order sentences were included since the events had a logical order even though they were described in reverse of that order. All responses to both logical and reverse logical order sentences were included, and responses to arbitrarily related sentences were excluded. A child received 1 point every time he dramatized the events in the clauses in their logical order of occurrence.

Examples of stimulus sentences and logical order responses to them appear below:

20. S Before the boy opened the door, he picked up the key.
$R$ (a) boy pick up key
(b) boy open door

21. S After the girl poured the syrup, she ate the pancakes.
$R$ (a) girl pour syrup
(b) girl eat pancakes

22. S The girl poured the soup before she opened the can.
$R$ (a) girl open can
(b) girl pour soup

23. S The boy cut the cake but before that he blew out the candles.

$\mathrm{R}$ (a) boy blow out candles (b) boy cut cake

\section{Order of Mention Strategy}

Responses to all sentences were included. A child received 1 point for every response in which he dramatized the clauses in the order in which they were mentioned. The following items illustrate this:

24. S The boy ate the hot dog when he opened the bottle.
$R$ (a) boy eat hot dog
(b) boy open bottle

25. S After the girl opened the bottle, she drank the pop.

$R$ (a) girl open bottle (b) girl drank pop

26. S The boy cut the cake but before that he blew out the candles.

$R \quad$ (a) boy cut cake boy blow out candles 


\section{Main Clause First Strategy}

If a child processes the main clause before the subordinate clause at least two outcomes are possible:

1. He interprets the event in the main clause as the first event and the event in the subordinate clause as the second event, dramatizing the main clause before the subordinate clause. A child was viewed as using this version of the main clause first strategy if in $75 \%$ or more of his responses he dramatized the main clause first and the subordinate clause second. This is the view of the main clause first strategy adopted in Coker (1978). This can be seen below:

27. S The girl poured the soup but before that she opened the can.
$\mathrm{R}$
(a) girl pour soup
(b) girl open can

28. S After the boy opened the door, he ate the hot dog.

$R \quad$ (a) boy eat hot dog (b) boy open door

2. The child forgets or ignores the subordinate clause, dramatizing only the main clause (French \& Brown, 1977; Johnson, 1975). If a child omitted the subordinate clause in $75 \%$ or more of his responses, he was regarded as using this version of the main clause strategy. In sum, then, each child's responses were examined for evidence that he used any of the above strategies. For the main clause strategy, each of the possible interpretations was tallied separately.

Our general analytic approach was to determine first whether a child's overall response patterns could be characterized by any of the preceding strategies. Then we explored the interaction between strategy use and clause logic. Hence, the $75 \%$ criterion was first applied to the sentences overall, then later separately to sentences in each of the three clause logic conditions. The frequency of strategy use by age for all sentences in all three logic conditions is summarized in Table III.

Using the $75 \%$ criterion levels, nine children used one interpretational strategy: three from Group I, three from Group II, two from Group III, and one from Group IV. So while strategy use was modest, it decreased with age. The logical order strategy was used by eight of these nine children; the order of mention was used by the remaining one. None of the children gave evidence of using a main clause first strategy according to either of the versions of this strategy. No child dramatized the main clause before the subordinate clause in $75 \%$ of his responses (view 1 of the main clause first strategy). The highest proportion of responses conforming to this was 63\% (32 out of 51 responses; four children at $61 \%$ and one child at $63 \%$ ). Four of these children were in 
Table III. Frequency of Major Strategy Use by Age ${ }^{a}$

\begin{tabular}{cccc}
\hline & \multicolumn{3}{c}{ Strategy } \\
\cline { 2 - 4 } Age group & Logical order & Order of mention & Main clause first ${ }^{b}$ \\
\hline I & 3 & 0 & 0 \\
II & 3 & 0 & 0 \\
III & 1 & 1 & 0 \\
IV & 1 & 0 & 0 \\
\hline
\end{tabular}

${ }^{a}$ Number of children at each age.

${ }^{b}$ Each main clause first analysis was computed separately.

Group I, one in Group III. Similarly, subordinate clauses were not omitted with any frequency (view 2). In fact, this was the least used response pattern. Sixteen children never omitted any subordinate clause and only one child omitted as many as 4 of 51 subordinate clauses.

Our final step was to examine the effect of clause logic on strategy use. We first examined the relationship between clause logic and the logical order strategy in its use in the reverse logical order condition. To understand this approach, consider the following. In the logical order condition, a child could correctly interpret a sentence such as 29 using any one of the several approaches: by dramatizing the events in the sequence to which he has become accustomed from his experience in the world, by relying on the meaning of the temporal connective, or a combination of both. Accordingly, evidence from the logical order condition alone is an insufficient test of his use of real-world knowledge in interpreting the sentence types tested here. In contrast, if a child relies solely on his knowledge of how events are related in the world to interpret a sentence such as 30 , he would always dramatize such events in reverse of the order described in the sentence. A reversal response to 30 would be girl open can, girl pour soup.

LOGICAL 29. The girl poured the soup after she opened the can. ORDER

REVERSE 30. The girl opened the can after she poured the soup. ORDER

As such, a consistent pattern of reversal responses in the reverse logical condition would be strong evidence that event relationship was used to the exclusion of other information in the sentence. If reversal responses were not consistently offered, it would suggest that children are utilizing 
Table IV. Clause Logic and Logical Order Strategy Use

\begin{tabular}{ccc}
\hline & \multicolumn{2}{c}{ Clause logic } \\
\cline { 2 - 3 } Age & Logical & Reverse logical \\
\hline I & 5 & 2 \\
I & 6 & 1 \\
III & 6 & 0 \\
IV & 6 & 1 \\
\hline
\end{tabular}

Table V. Clause Logic and Order of Mention Strategy Use ${ }^{a}$

\begin{tabular}{cccc}
\hline & \multicolumn{3}{c}{ Clause logic } \\
\cline { 2 - 4 } Age group & Logical & Reverse logical & Arbitrary \\
\hline I & 0 & 0 & 2 \\
II & 1 & $3^{b}$ & 1 \\
III & 0 & 1 & $2^{c}$ \\
IV & 0 & 0 & 0 \\
\hline
\end{tabular}

${ }^{a}$ Number of children employing the order of mention strategy in each clause logic condition. ${ }^{b}$ One child in Group II used this strategy in both the Logical Order and Reverse Logical conditions.

${ }^{c}$ One child in Group III used this strategy in both the Reverse Logical and Arbitrary conditions.

both clause logic and the meaning of the temporal connective. Responses to sentences in the arbitrary condition were excluded from this analysis because the events described do not occur in a predictable order. These results are summarized in Table IV.

The logical order strategy was applied regularly at all ages in the logical order condition only; it occurred seldom in the reverse logical order condition, which was the more stringent test of its use. This suggests that while some children and more at the younger ages may rely solely on their knowledge of predictable event patterns to interpret sentences (as can be seen in Table IV, Reverse Logic condition), most seem to utilize the meaning of the temporal connective too.

Next we examined the use of the order of mention strategy in the three clause logic conditions. This can be seen in Table V. Eight different children (two in Group I, four in Group II, and two in Group III) used an order of mention strategy as their modal response in at least one of the 
Table VI. Main Clause First Strategy ${ }^{a}$ and Clause Logic

\begin{tabular}{cccc}
\hline & \multicolumn{3}{c}{ Clause logic } \\
\cline { 2 - 4 } Age group & Logical & Reverse logical & Arbitrary \\
\hline I & 0 & 1 & 0 \\
II & 0 & 2 & 0 \\
III & 0 & 0 & 0 \\
IV & 0 & 1 & 0 \\
\hline
\end{tabular}

${ }^{a}$ Version 1 (dramatize main clause first, subordinate clause second).

clause logic conditions. Use of this strategy occurred least often in the logical condition, where the clause context could be used to correctly interpret the sentences. No child in Group IV applied this strategy to interpret the test sentences.

Last, we explored whether the main clause first strategy varied with clause logic even though no child used this as a general interpretational heuristic. We did not consider version 2 (omission of the subordinate clause) since the maximum number of subordinate clauses omitted over all three clause conditions was four. As for version 1 (main clause dramatized first, subordinate clause second), no children employed this in response either to logically or arbitrarily related clauses. Four children used it in the reverse logical clause condition, although no clear age-related pattern was evident. This is summarized in Table VI.

\section{DISCUSSION}

\section{Clause Logic}

The role of clause logic reported here is consistent with previous findings (French \& Brown, 1977; Kavanaugh, 1979). Children generally were more successful at interpreting sentences where the clause context reinforced interpretation of the temporal connectives (logical condition) than where it did not (reverse logical and arbitrary). In fact, in the reverse logical condition where the connective was at odds with the clause context, children had the greatest difficulty understanding the test sentences. This was true in Groups I, II, and IV; in Group III the proportion of correct responses to arbitrary and reverse logical sequences was nearly equal.

Clause context also was found to be important in determining the relative order in which particular temporal connectives are acquired and 
in helping a child decide between the senses of a temporally ambiguous term. Contextually supported terms, before and after were learned earlier than while. (This is supported by Feagans, 1980.) Similarly, the sense of when that can be discovered from clause relationship ("after") is learned earlier than the unsupported sense ("while").

At 3 years of age we begin to see evidence that clause context influences the interpretation of multiple-sense lexical items, and that by age 5 this effect is stronger. Recall that "after" interpretations of when generally were more commonplace in the logical as against the arbitrary condition. However, since while responses were so infrequent at that age, it could not be claimed that children chose between alternate senses of when based on context. Rather, by 5 years of age, "while" readings of when begin to appear with some regularity in both the logical and arbitrary conditions, but more so in the latter. This suggests that clause content is now playing a more important role in choosing between alternate senses of a lexical item. Information from more children would be necessary to clarify this picture, as would data from a range of multiple-sense terms.

Although clause logic was found to play a role, it does not account for many of the responses here. The finding that clause context assisted logical order but not reverse logical order sequences suggests that even at the ages studied, children are not relying solely on clause context for their interpretation of temporal terms. This is consistent with Stroehner and Nelson (1974), who report a developmental shift from extralinguistic strategies such as event probability to the syntactic interpretation of sentences. (See also Bever, 1970; Cromer, 1976).

If children were relying solely or primarily on clause context, we would have found them reversing the reverse logical events-e.g., The girl blew out the candles after she cut the cake dramatized as (1) girl blow out candles, (2) girl cut cake. Instead we saw that only 6 of 16 children seemed to rely primarily on clause context for their interpretation (Table III). The rest were apparently including some interpretation of the temporal connective too. So while clause context plays a role in the first few years of life, by 3 years of age children are taking into account other types of information in their emerging views of word meaning. This study leaves open what that information is, how children coordinate and integrate the data they collect about words, and the form in which they represent it.

One alternate interpretation of the role of clause context reported here is that it would have been difficult for the children to perform some actions in reverse of their logical order-for example, pouring the soup 
before opening the can. Hence, the logical order was the result of behavioral biases. This potential confounding was taken into account in task design. As part of the instructions and preliminary rehearsal prior to the test items, children were shown how to dramatize the stimulus events and these dramatizations were symbolic. The soup can was not actually opened; rather each child was shown how to move the doll's hand as though he/she were opening the can. ${ }^{3}$ All children expressed delight at this training and none displayed any difficulty dramatizing the events. As such, physical difficulty cannot satisfactorily explain the pattern of results observed here.

\section{Interpretational Strategies}

The role of clause logic reported here extends our view of the contribution of interpretational strategies to the acquisition of temporal connectives. Twenty-eight percent (9) of the children studied used a nonlinguistic strategy to respond to the test sentences. Eight of these adopted the logical order strategy, dramatizing the sentences according to their knowledge of how such events are sequenced in the real world. Two previously reported strategies-order of mention and main clause firstwere found to play at most a secondary role in interpreting sentences with temporal connectives. When all logic conditions were taken together, only one child used the order of mention strategy. None used either version of the main clause first strategy. Omissions of subordinate clause events were negligible. Furthermore, no child adopted a general strategy of dramatizing the main clause first and the subordinate clause second. When clause logic was taken into account a somewhat more complex picture emerged. Neither the order of mention or main clause strategies was applied routinely to sentences in the logical order condition. The higher rate of strategy use reported elsewhere (Clark, 1971; Amidon \& Carey, 1972) would seem to be a function of the limited range of temporal connectives studied and the characteristics of the tasks (Johnson, 1975).

In previous work, it was suggested that the variation among studies in the omission rates of subordinate clauses that, as will be recalled, was evidence for the main clause strategy, may arise from task differences. Johnson (1975) found that omissions were greater in response to

\footnotetext{
${ }^{3}$ Undoubtedly these were like a good deal of the dramatic play in which preschool children spontaneously engage.
} 
imperatives (e.g., Amidon \& Carey, 1972) than to nonimperatives (e.g., Clark, 1971). In the present investigation, the low rate of omissions may also have been due to the type of sentences used. Since many of the sentences described logically related events, children may have developed a mental set toward relating pairs of events. Even so, much of the language children hear in their early years describes these very sorts of events and therefore it would seem likely that children pay attention to both events in a sentence under normal conditions.

Although this study provides clues to the contribution of clause context, it does not provide evidence on what sorts of evidence children need in order to refine their interpretations of temporal terms. Specifically, what types of experiences could draw their attention to the limitations on the logical order strategy? We know that a good deal of children's experience is with routine, often ritualized patterns that undoubtedly reinforce hypotheses based on probability. They know that they have their diapers changed before sleep and their food cut up before eating. One possible input to this process of refining probability-based hypotheses would be adult behaviors that direct a child's attention to the unexpected or nonroutine, making it more salient. This might consist of gestural indicators to the unexpected or might include linguistic strings that contrast the unexpected with the routine, "He did it after, not before", perhaps reinforced by emphatic stress. By highlighting violations of norm, adults may help draw children's attention to the data needed to modify their hypotheses about word meaning. As children grow older, they are exposed to greater environmental variation, and this too provides not only experience with deviations from the routine but talk about these deviations. This line of inquiry would then be part of a more general exploration of how children make the transition from nonlinguistic to linguistically based hypotheses. This is to say that we will need to seek a more comprehensive picture of the types of language-based information to which children begin to attend, such as the kinds of syntactic detail that contribute to meaning, in order to understand more fully how temporal connectives are acquired.

\section{REFERENCES}

Amidon, A., \& Carey, P. (1972). Why five-year-olds cannot understand before and after. Journal of Verbal Learning and Verbal Behavior, 11, 417-423.

Bever, T. (1970). The comprehension and memory of sentences with temporal relations. In G.B. Flores d'Arcais \& W.J.M. Levelt(Eds.), Advances in psycholinguistics. Amsterdam/ New York: North-Holland/American Elsevier. 
Clark, E.V. (1971). On the acquisition of the meaning of before and after. Journal of Verbal Learning and Verbal Behavior, 10, 266-275.

Coker, P.L. (1978). Syntactic and semantic factors in the acquisition of before and after. Journal of Child Language, 5, 261-277.

Cromer, R. (1976). Developmental strategies for language. In V. Hamilton \& M.D. Vernon (Eds.), The development of cognitive processes. London: Academic Press.

Feagans, D.L. (1980). Children's understanding of some temporal terms denoting order, duration, and simultaneity. Journal of Psycholinguistic Research, 9, 41-57.

Ferreiro, E. (1971). Les relations temporelles dans le language de l' enfant. Geneva: Libraire Droz.

French, L.A., \& Brown, A.L. (1977). Comprehension of before and after in logical and arbitrary sequences. Journal of Child Language, 4, 247-256.

Johnson, H.L. (1975). The meaning of before and after for preschool children. Journal of Experimental Child Psychology, 19, 88-99.

Kavanaugh, R.D. (1979). Observations on the role of logically constrained sentences in the comprehension of before and after. Journal of Child Language, 6, 353-357.

Keller-Cohen, D. (1974). The acquisition of temporal connectives in pre-school children. Unpublished doctoral dissertation, State University of New York at Buffalo.

Stroehner, H., \& Nelson, K. (1974). The young child's development of sentence comprehension: Influence of event probability, non-verbal context, syntactic form, and strategies. Child Development, 45, 567-576.

Trosborg, A. (1982), Children's comprehension of "before" and "after" reinvestigated. Journal of Child Language, 9, 381-402. 